\title{
Effects of changing from a diet with saturated fat to a diet with $n-6$ polyunsaturated fat on the serum metabolome in relation to cardiovascular disease risk factors
}

\author{
Kristina Pigsborg ${ }^{1}$ [D $\cdot$ Gözde Gürdeniz ${ }^{2}$ O Oscar Daniel Rangel-Huerta ${ }^{3} \cdot$ Kirsten B. Holven $^{3,4} \cdot$ Lars Ove Dragsted $^{1}$. \\ Stine M. Ulven ${ }^{3}$
}

Received: 17 August 2021 / Accepted: 21 December 2021 / Published online: 9 January 2022

(c) The Author(s) 2022

\begin{abstract}
Purpose Replacing saturated fatty acids (SFA) with polyunsaturated fatty acids (PUFA) is associated with a reduced risk of cardiovascular disease. Yet, the changes in the serum metabolome after this replacement is not well known. Therefore, the present study aims to identify the metabolites differentiating diets where six energy percentage SFA is replaced with PUFA and to elucidate the association of dietary metabolites with cardiometabolic risk markers.

Methods In an 8-week, double-blind, randomized, controlled trial, 99 moderately hyper-cholesterolemic adults (25-70 years) were assigned to a control diet (C-diet) or an experimental diet (Ex-diet). Both groups received commercially available food items with different fatty acid compositions. In the Ex-diet group, products were given where SFA was replaced mostly with $n$-6 PUFA. Fasting serum samples were analysed by untargeted ultra-performance liquid chromatography high-resolution mass spectrometry (UPLC-HRMS). Pre-processed data were analysed by double cross-validated Partial Least-Squares Discriminant Analysis (PLS-DA) to detect features differentiating the two diet groups.

Results PLS-DA differentiated the metabolic profiles of the Ex-diet and the C-diet groups with an area under the curve of 0.83. The Ex-diet group showed higher levels of unsaturated phosphatidylcholine plasmalogens, an unsaturated acylcarnitine, and a secondary bile acid. The C-diet group was characterized by odd-numbered phospholipids and a saturated acylcarnitine. The Principal Component analysis scores of the serum metabolic profiles characterizing the diets were significantly associated with low-density lipoprotein cholesterol, total cholesterol, and triglyceride levels but not with glycaemia.

Conclusion The serum metabolic profiles confirmed the compliance of the participants based on their diet-specific metabolome after replacing SFA with mostly $n-6$ PUFA. The participants' metabolic profiles in response to the change in diet were associated with cardiovascular disease risk markers. This study was registered at clinicaltrials.gov as NCT 01679496 on September 6th 2012.
\end{abstract}

Keywords Cardiovascular risk markers $\cdot$ Nordic diet $\cdot$ Fatty acids $\cdot$ Phospholipids $\cdot$ Metabolomics

Kristina Pigsborg

kpj@ nexs.ku.dk

1 Department of Nutrition, Exercise and Sports, Faculty of Science, University of Copenhagen, Rolighedsvej 26, 1958 Frederiksberg, Denmark

2 Department of Food Science, University of Copenhagen, Frederiksberg, Denmark

3 Department of Nutrition, Institute for Basic Medical Sciences, University of Oslo, Oslo, Norway

4 Norwegian National Advisory Unit On Familial Hypercholesterolemia, Department of Endocrinology, Morbid Obesity and Preventive Medicine, Oslo University Hospital Aker, Nydalen, PO Box 4959, 0424 Oslo, Norway

\section{Introduction}

Cardiovascular disease (CVD) is still one of the leading contributors to death worldwide [1]. Many risk markers associated with CVD can be modified and especially fatty acids (FAs) are suggested to have a central role affecting lowdensity lipoprotein (LDL) cholesterol levels [2, 3]. Based on strong evidence, general dietary recommendations and WHO guidelines suggest that replacing saturated fatty acids (SFA) with polyunsaturated fatty acids (PUFA) reduces the risk of CVD by decreasing LDL-cholesterol levels [3-5]. Despite this solid evidence, it has been suggested that foods 
rich in SFA may contribute differently to health, depending on the food source $[6,7]$.

The "Healthy Nordic Diet" (HND) is based on the Nordic Nutrition Recommendations [8-10] and one of the characteristics of HND compared to the average habitual Nordic diet is the source and type of fat. In an HND, a higher amount of dietary fat derives from plant sources, such as rapeseed oil and sunflower oil rich in $n-6$ PUFAs instead of dairy products rich in SFAs.

The health effects of an HND have previously been investigated in different Nordic population groups, and beneficial effects of HND on blood lipids among subjects at risk of CVD were reported [2, 10-14]. However, changing the whole diet can be challenging to enforce, and therefore, we performed a randomized controlled trial in Norway, exploring the effect of a Nordic diet with a focus primarily on fat quality, replacing SFA with PUFA [15]. We have previously reported the primary outcome of this study, which showed a reduction in total cholesterol and LDL cholesterol by exchanging only a few commercials, regularly consumed food items in the diet (15). In addition, using a metabolic profiling approach, we showed that a large number of lipoprotein subclasses, myristoyl- and palmitoylcarnitines, and kynurenine were reduced, while bile acids, proprotein convertase subtilisin/kexin type 9 , acetate, and acetoacetate were increased in blood when dietary SFA was replaced with mostly $n$-6 PUFA [16].

By utilizing the comprehensive coverage of LC-MSbased untargeted metabolomics, it is possible to reveal alterations in metabolism promoting the development of CVD and to identify novel biomarkers for cardiometabolic disease $[17,18]$. To our knowledge, this would be the first untargeted metabolomics study exploring the role of fat quality, and not the whole diet, as earlier studies have focused on [19-21].

The main aim of this sub-study is to identify the metabolic profiles differentiating diets with different fatty acid compositions using untargeted metabolomics, to elucidate the association of diet-related metabolite patterns with cardiometabolic risk markers, and to support further understanding of the health beneficial mechanisms after replacing SFA with mostly $n-6$ PUFA.

\section{Methods}

\section{Study design and subjects}

An 8-week, double-blind, randomized, controlled dietary intervention study was conducted at the Oslo and Akershus University College of Applied Sciences and the University of Oslo, Norway, from July 2012 until April 2014. Details of the study design and description of participants have been described previously [15]. In short, 99 healthy adults (58\% females) aged 25-70 years with LDL-cholesterol $\geq 3.5 \mathrm{mmol} / \mathrm{L}$, total cholesterol within the normal range for each age group, and triglyceride (TG) $\leq 2.6 \mathrm{mmol} / \mathrm{L}$ participated in this study. Included study subjects had BMI between 20 and $35 \mathrm{~kg} / \mathrm{m}^{2}$, and had a stable body weight the last three months prior to inclusion. The study subjects were excluded if they used lipid-lowering drugs.

Over a 2-week run-in period, the control food items were included in all subjects' daily diets. Afterwards, subjects were randomly assigned 1:1 stratified by age and gender to a control diet (C-diet) group that included the control food items in their diet and an experimental diet (Ex-diet) group that included experimental food items.

The experimental food items were the same type of products as the control food items, but with an altered fatty acid composition (SFAs replaced with mostly $n-6$ PUFAs). The food items in the C-diet group or the Ex-diet group were butter-based spread or margarine-based spread, butter or liquid margarine, and olive oil or rapeseed and sunflower oil, respectively. Likewise, products, such as liver paté, cheese, bread, muesli cereals, cream, mayonnaise, and crème fraîche, were given to the study subjects in the C-diet group, and some of the SFAs were replaced with rapeseed and sunflower oils in the products for the Ex-diet group. More information on the study food items is described in detail earlier [15]. The participants were instructed to include these food items in their daily diet and otherwise continue to eat as normal. The participants included the food items in their daily diet and the products were mainly used for breakfast, lunch, and supper, and oils, butter/margarine, cream, and crème fraîche were used for cooking. Therefore, we do believe that the foods item mainly were used to freshly prepare their meals at home. Based on the minimum intake of the food items, the $n-6$ PUFA intakes (mainly linoleic acid) were $4.2 \mathrm{~g} / \mathrm{d}$ and $12.9 \mathrm{~g} / \mathrm{d}$, the $n-3$ PUFA intakes (mainly $\alpha$-linolenic acid) were $0.9 \mathrm{~g} / \mathrm{d}$ and $1.5 \mathrm{~g} / \mathrm{d}$ and the SFA intakes were 19.2 and $5.7 \mathrm{~g} / \mathrm{d}$ for the $\mathrm{C}$-diet and Ex-diet group, respectively, during the intervention. More information on the FAs composition of the food items is provided earlier [15]. Dietary intake was registered during the intervention, and all subjects completed a fourday pre-coded food diary which also included a validated photography booklet with four different portion sizes at the beginning and the end of the intervention period [15].

\section{Sample preparation and untargeted UPLC-QTOF-MS profiling}

Clinical and blood laboratory assessments were performed at baseline and after 8 weeks of follow-up. The day before blood sampling, the subjects were told to refrain from alcohol consumption and vigorous physical activity. Venous blood samples were drawn after an overnight fast $(\geq 12 \mathrm{~h})$. 
Serum was obtained from silica gel tubes (Becton Dickinson Vacutainer Systems) and kept at room temperature for $\leq 30$ min until centrifugation $(1500 \times \mathrm{g}, 15 \mathrm{~min})$. Fasting serum concentrations of total cholesterol, LDL cholesterol, HDL cholesterol, TG, glucose, and insulin were measured by standard methods at a routine laboratory (Fürst Medical Laboratory) as previously described [15].

As described in detail [22], serum protein precipitation was performed before serum samples were analysed by an ultra-performance liquid chromatography (UPLC) system coupled to a quadrupole time-of-flight mass spectrometer (Premier QTOF-MS, Waters Corporation, Manchester, UK). The sample analysis was based on previously described analytical methodologies [23]. Briefly, serum samples were placed in 96-well Sirocco ${ }^{\mathrm{TM}}$ plates and five $\mu \mathrm{L}$ aliquots of each sample were injected into an Acquity UHPLC (Waters) equipped with an HSS T3 C18 column $(2.1 \times 100 \mathrm{~mm}, 1.8 \mu \mathrm{m})$ coupled with an HSS T3 $\mathrm{C} 18$ precolumn $(2.1 \times 5 \mathrm{~mm}, 1.8 \mu \mathrm{m})$ (Waters), using a gradient operated for $10.0 \mathrm{~min}$. Experimental conditions throughout the analysis are shown in Table 1SI. Blanks consisting of $0.1 \%$ formic acid in $\mathrm{H}_{2} 0$, external metabolomics standard mixtures, and a pooled sample (of each analytical batch) were injected after every 15 samples and at the end of each analytical batch.

\section{Data pre-processing and pre-treatment}

The raw data from the LC-MS was converted to an intermediate.netCDF format with the DataBridgeTM utility provided with the MassLynx software (Waters). The data acquired in positive and negative ionization modes were preprocessed separately with MZmine (ver. 2.31) [24]. The optimized preprocessing parameters are given in the online supplementary information (Table 2SI). Subsequently, the pre-processed data were imported to the software program, Matlab R2017b (ver. 9.3.0; Mathworks Inc., MA, US). Feature filtering was applied: (1) the features present in blanks were removed, (2) features detected before $0.3 \mathrm{~min}$ and later than $9.42 \mathrm{~min}$ were excluded, (3) early (0.3-0.8 $\mathrm{min})$ features having 4-9 as the first decimal place of the mass-to-charge ratio $(\mathrm{m} / \mathrm{z})$ were deemed implausible and removed, and (4) potential isotopes along with duplicates were excluded.

Intensities were corrected for inter-batch variation; the intensity of each feature detected in the samples in each batch was multiplied by the batch average and divided by the overall average across all batches [22]. Furthermore, intra-batch drift was corrected using the online software program Galaxy (ver. 2.1.2). Afterwards, features were grouped within a retention time (RT) range of $0.01 \mathrm{~min}$ if their correlation coefficients were higher than 0.7. Lastly, features from negative and positive modes were concatenated into a single final data set.

\section{Statistical analysis}

The sample size of this study was calculated based on the primary outcome as published previously [15]. Power calculations estimated that 180 subjects (including a 20\% dropout rate) were required to obtain $80 \%$ power with a type I error of $5 \%$ to detect a difference of $8 \%$ in LDL cholesterol concentrations at the end of the study between the two groups. Post hoc analyses showed that the number of subjects recruited gave sufficient power with the observed $10 \%$ change in LDL cholesterol between the groups $(n=47$ subjects in each group). Prespecified secondary outcomes were other lipids, inflammatory markers, metabolomics, and transcriptomics.

Data analysis was performed in Matlab® using PLStoolbox (ver. 8.7, Eigenvector Research, MA, US) with baseline-corrected data (serum baseline samples subtracted from 8-week sample) to reduce the variations in the metabolic profile caused by individuality.

Firstly, principal component analysis (PCA) was applied to explore for grouping patterns, and secondly, partial leastsquares discriminant analysis (PLS-DA) models was developed to identify the most differentiating features separating the Ex-diet and C-diet group. Double cross-validation was applied for PLS-DA model evaluation [25]. Briefly, $80 \%$ of the data were used as a training set and the remaining $20 \%$ was used as a test set. The samples in the training set were cross-validated to find the optimal complexity of the model (number of latent variables). The minimum numbers of latent variables providing the lowest cross-validation $(\mathrm{CV})$ classification errors for the model were chosen and variables that had the lowest influence based on variable importance in projection (VIP) plots were removed. The model was then recalculated based on the reduced number of variables in the training set. This continued as long as the selected features improved the model performance by decreasing the $\mathrm{CV}$ classification error in the training set. The performance of the selected features in terms of differentiating the samples from the Ex-diet and the C-diet group was based on the rate of misclassification errors (ER) and area under the curve (AUC) of the test set samples. One hundred PLS-DA models with randomly selected training/test set pairs were generated and a mean of the test set ER and a mean of the AUC were calculated to evaluate the performance of the models and the final number of included features. Next, features present in $>70 \%$ of the models (from 100 training/test set pairs) were selected for the final feature set.

The associations between diet-specific serum metabolic profiles reflecting the diets and the clinical outcomes of interest, i.e. total cholesterol, TG, LDL cholesterol, HDL cholesterol, glucose, and insulin, were explored by linear models applied using $\mathrm{R}$ (ver. 3.6.1) [26]. The metabolic profiles were characterized by the selected features from the 
PLS-DA and translated into principal component scores for comparison with the clinical outcomes. Finally, causal mediation analysis between the metabolic profile scores and the clinical outcomes was performed using the mediation package [27] to explore if the altered metabolome as a mediator of the effect of the intervention on clinical outcomes, i.e. total cholesterol, TG, LDL-cholesterol, HDL-cholesterol, and glucose.

\section{Metabolite identification}

Unknown compounds with a signal area below 100 counts were excluded from further identification as MS/MS experiments are not feasible with such low intensities (Table 3SI). For unknown compounds with higher intensity than 100, MS/MS spectra (ionized with either 10,20 , and $30 \mathrm{eV}$ or 20,36 , and $48 \mathrm{eV}$ using argon/nitrogen) were recorded with a tandem mass spectrometer (VION from Waters) using the same prior UPLC separation as described above (Table 4SI). MS/MS spectra were compared with available databases, such as METLIN (https://metlin.scripps.edu) [28], Human Metabolome Database (HMDB) (http://www.hmdb.ca) [29], and produced by in silico fragmentation tools such as CFMID (http://cfmid.wishartlab.com/) [30], FingerID (https:// www.csi-fingerid.uni-jena.de/) [31] and LIPID MAPS ${ }^{\mathrm{R}}$ (http://www.lipidmaps.org) [32].

Authentic standards (Table 5SI) and the sample that contains the metabolites of interest most abundantly were run consecutively on the same platform for correct annotation. Furthermore, an algorithm was used to predict retention times of homologous series of phospholipids based on their physicochemical properties and their orthogonal data as described previously [33]. Prediction plots for lysophosphatidylcholines (lysoPCs) and PCs are shown in supplementary information Figs. 1SI and 2SI. With increasing numbers of carbon atoms in the fatty acyl chain in PCs and lysoPCs polarity decreases, resulting in a stepwise increase in retention time. Likewise, an increase of double bonds reduces the retention time in a predictable manner [33]. For several of the phospholipids, it was possible to detect both isomers with the different fatty acyl chains located in either sn 1 or sn2 position. Authentic standards were used to validate the prediction plots.

The identified metabolites were classified with a level of identification I-V in accordance with the Metabolomics Standards Initiative classification scheme [34]. Examples of structure elucidations of level II identified compounds are provided in supplementary information Fig. 3SI.

\section{Results}

Ninety-nine study subjects provided serum samples both at baseline and at the end of the intervention. As reported previously, the Ex-diet group had a reduction in LDL-cholesterol compared to the $\mathrm{C}$-diet group $(\mathrm{P}<0.001)$ after the 8 -week intervention [15]. Moreover, reductions from baseline to end of intervention of total cholesterol, HDL cholesterol, and TG levels were also observed between the Ex-diet and the $\mathrm{C}$-diet group. Other baseline characteristics of the participants of the two groups are shown in Table 1 together with the changes over time for the clinical outcomes. Dietary intake during the intervention has been reported in the original publication of this [15]. In short, the intake of energy was similar in the two groups, but the energy percent intake of protein, PUFA, and fibre was significantly higher in the

Table 1 Baseline characteristics and biochemical measurements from baseline and end of the study

\begin{tabular}{|c|c|c|c|c|c|}
\hline & \multicolumn{2}{|l|}{ C-diet $(n=52)$} & \multicolumn{2}{|l|}{ Ex-diet $(n=47)$} & \multirow[t]{2}{*}{$P$ values ${ }^{\dagger}$} \\
\hline & Baseline & End of study & Baseline & End of study & \\
\hline Age, $y$ & $55.2 \pm 9.8$ & - & $53.6 \pm 9.7$ & - & \\
\hline Sex, $n$ females $(\%)$ & $31(59.6)$ & - & $27(57.4)$ & - & \\
\hline Body mass index, $\mathrm{kg} / \mathrm{m}^{2}$ & $24.6 \pm 3.0$ & $24.8 \pm 3.06^{*}$ & $25.4 \pm 2.9$ & $25.4 \pm 2.9$ & 0.043 \\
\hline LDL-cholesterol, mmol/L & $4.1 \pm 0.6$ & $4.2 \pm 0.6$ & $4.2 \pm 0.6$ & $3.8 \pm 0.5^{*}$ & $<0001$ \\
\hline HDL-cholesterol, mmol/L & $1.7 \pm 0.4$ & $1.7 \pm 0.5$ & $1.7 \pm 0.5$ & $1.6 \pm 0.4^{*}$ & 0.005 \\
\hline Total-cholesterol, $\mathrm{mmol} / \mathrm{L}$ & $6.6 \pm 0.8$ & $6.6 \pm 0.8$ & $6.6 \pm 0.8$ & $6.0 \pm 0.7^{*}$ & $<0.001$ \\
\hline Triglyceride, $\mathrm{mmol} / \mathrm{L}$ & $1.20(0.92-1.48)$ & $1.24(1.01-1.60)^{*}$ & $1.31(0.87-1.56)$ & $1.19(0.77-1.46)$ & 0.002 \\
\hline Glucose, mmol/L & $5.2(4.9-5.4)$ & $5.2(5.1-5.4)$ & $5.3(5.0-5.6)$ & $5.3(5.0-5.6)$ & 0.366 \\
\hline Insulin, $\mathrm{pmol} / \mathrm{L}$ & $43(31-60)$ & $43(33-72)$ & $52(36-79)$ & $58(42-73)$ & 0.961 \\
\hline $\mathrm{HbA} 1 \mathrm{c}, \%$ & $5.3(5.2-5.5)$ & $5.4(5.2-5.6)$ & $5.3(5.2-5.5)$ & $5.3(5.2-5.4)$ & 0.724 \\
\hline
\end{tabular}

Data are provided as mean \pm SD or as median (IQR). ${ }^{*}$ Significant difference from baseline to end of the study tested with paired $t$ test or Wilcoxon's signed-rank test

${ }^{\dagger}$ Difference between groups tested with independent $t$ test or Mann-Whitney $U$ test

$L D L$ low-density lipoprotein; $H D L$ high-density lipoprotein; $H b A l c$ hemoglobin A1c 
Ex-diet group compared to the C-diet group (16.5 E\% versus $15.0 \mathrm{E} \%$ of protein, $12.0 \mathrm{E} \%$ versus $5.6 \mathrm{E} \%$ of PUFA, and $37.2 \mathrm{~g}$ versus $25.7 \mathrm{~g}$ of fibre, respectively). On the other hand, the energy percent intake of SFA and carbohydrates was significantly higher in the C-diet group compared to the Ex-diet group (18.0 E\% versus 11.5 E\% of SFA and 36.6 E\% versus $34.2 \mathrm{E} \%$ of carbohydrates, respectively). In addition, the intake of vitamin A, retinol, and alpha-tocopherol was significantly higher in the C-diet group compared to the Exdiet group, and the intake of vitamin D and thiamine was significantly higher in the Ex-diet group compared to the C-diet group (Supplementary Table 6SI).

\section{Metabolic profiles reflecting the Ex-diet vs. C-diet}

The data pre-processing and further data filtering resulted in 740 and 2120 features for negative and positive modes, respectively. The final PLS-DA model performed well in terms of differentiating samples from the Ex-diet and C-diet groups with an average AUC and ER of 0.83 and 0.23 , respectively. The result of the variable selection process provided 32 features that were present in at least $70 \%$ of the models (with 100 training/test pairs). A PCA based on these metabolites shows that the first two principal components explain $43.2 \%$ of the variation with good separation between the two groups (Fig. 1). The identified metabolites are presented in Table 2 together with their retention time, observed mass, annotation, and observed fragments from the MS/MS experiments. Separation and box plots of each of the metabolites are listed in supplementary information Table 7SI.

The serum metabolic pattern mainly reflects the different lipid compositions of the two diets. Among the selected metabolites, the Ex-diet group was dominated by unsaturated plasmalogens, e.g. PC(P-38:4) (Fig. 1). Furthermore, an unsaturated medium-chain carnitine, e.g. C8:1-carnitine, together with the bile acids lithocholic acid (LCA) and indolelactic acid (ILA) also characterized the Ex-diet group. In contrast, the $\mathrm{C}$-diet group was mainly characterized by odd-numbered phospholipids, e.g. PC(31:0), PC(33:0), and $\mathrm{PC}(33: 1)$. The C-diet group also had increased levels of the lysophospholipid, lysoPC(14:0), representing myristic acid present in butter and milk. Furthermore, a saturated medium-chain carnitine, C9:0-carnitine, and the short-term glycaemic marker, 1,5-anhydroglucitol (1,5-AG) (same as 1,5-anhydrosorbitol), also characterized the C-diet group.

From a hierarchical cluster analysis using Spearman correlations between the selected metabolites from the final PLS-DA model, associations between the compounds are shown in Fig. 2. The metabolites differentiating the two

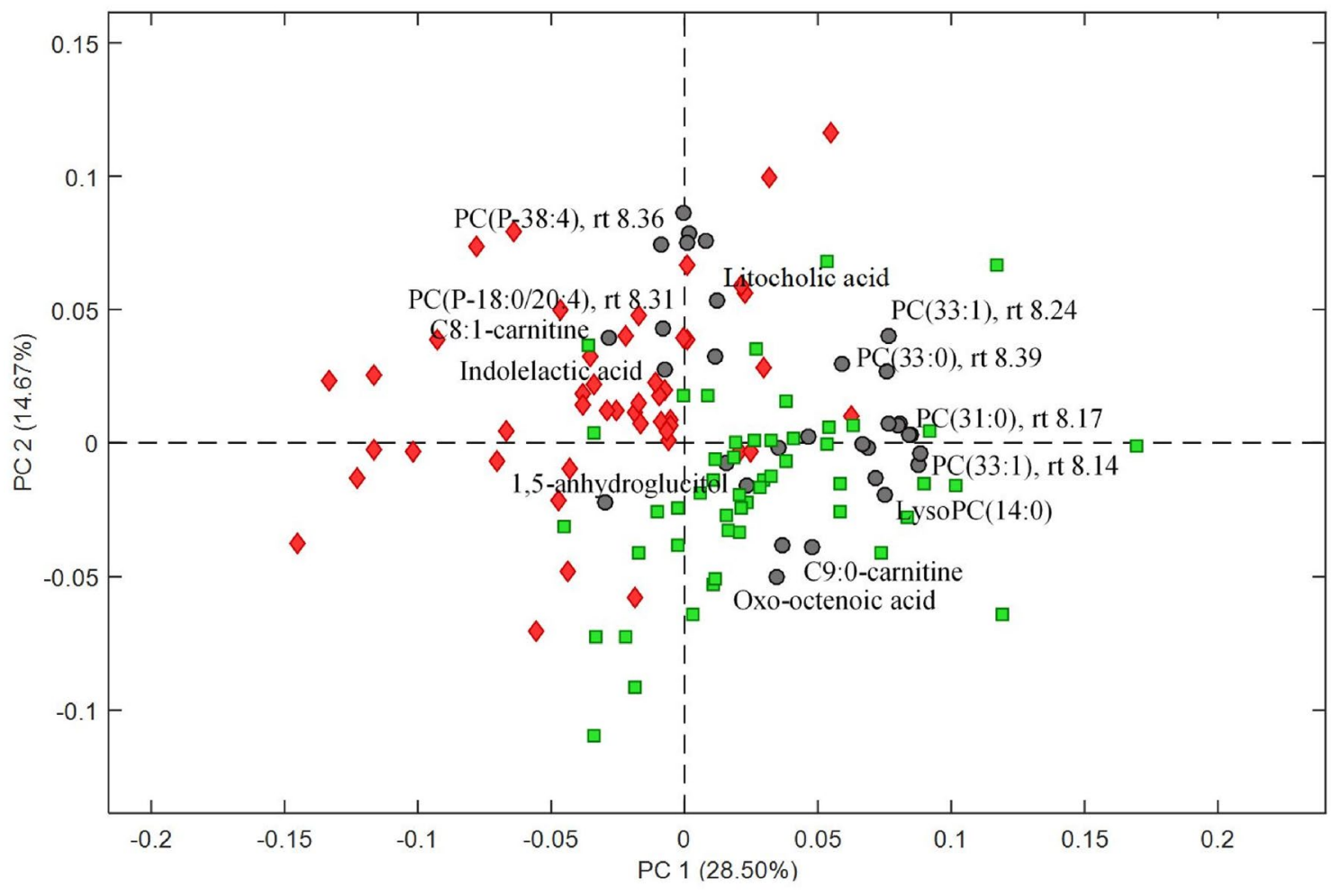

Fig. 1 PC1 vs. PC2 biplot based on features from the final PLS-DA model (AUC $=0.83$, classification error $=0.23$ ) for diet group separations. Subjects are coloured according to diet (red diamonds for Ex- diet and green squares for $\mathrm{C}$-diet) and identified metabolites for the diets are highlighted (grey points), $P C(P)$ phosphatidylcholine plasmalogen, $P C$ phosphatidylcholine, $R T$ retention time 
Table 2 Identified serum metabolites higher after diets rich in polyunsaturated fatty acid (Ex-diet) or saturated fatty acids (C-diet)

\begin{tabular}{|c|c|c|c|c|c|}
\hline $\begin{array}{l}\text { Suggested annotation/ } \\
\text { Level of identification }\end{array}$ & $\mathrm{RT}(\min )$ & Observed $\mathrm{m} / \mathrm{z}$ & Suggested ion & $\begin{array}{l}\text { Fragments and } \\
\text { adducts }\end{array}$ & Diet \\
\hline Indolelactic acid ${ }^{\mathrm{I}}$ & 5.73 & 204.0690 & {$[\mathrm{M}-\mathrm{H}]^{+}$} & & Ex-diet \\
\hline C8:1-carnitine ${ }^{\mathrm{II}}$ & 5.77 & 286.1990 & {$[\mathrm{M}+\mathrm{H}]^{+}$} & $\begin{array}{l}227.14 \\
144.11 \\
125.10 \\
85.03 \\
43.06\end{array}$ & Ex-diet \\
\hline Lithocholic acid ${ }^{\mathrm{I}}$ & 7.30 & 445.2947 & {$[\mathrm{M}+\mathrm{HCOONa}]^{+}$} & $\begin{array}{l}399.23 \\
377.24 \\
359.25 \\
73.03\end{array}$ & Ex-diet \\
\hline $\mathrm{PC}(\mathrm{P}-18: 0 / 20: 4)^{\mathrm{I}^{*}}$ & 8.31 & 794.5939 & {$[\mathrm{M}+\mathrm{H}]^{+}$} & $\begin{array}{l}510.38 \\
184.08 \\
86.10\end{array}$ & Ex-diet \\
\hline PC(P-20:4/18:0) ${ }^{\mathrm{III}}$ & 8.36 & 794.6068 & {$[\mathrm{M}+\mathrm{H}]^{+}$} & $\begin{array}{l}510.38 \\
184.08 \\
86.10\end{array}$ & Ex-diet \\
\hline 1,5-anhydroglucitol & 0.69 & 187.0588 & {$[\mathrm{M}+\mathrm{Na}]^{+}$} & & C-diet \\
\hline C9:0-carnitine ${ }^{\mathrm{II}}$ & 6.15 & 302.2329 & {$[\mathrm{M}+\mathrm{H}]^{+}$} & $\begin{array}{l}243.17 \\
159.15 \\
144.11 \\
85.03 \\
43.06\end{array}$ & C-diet \\
\hline Oxo-octanoic acid ${ }^{\text {III }}$ & 6.28 & 155.0697 & {$[\mathrm{M}-\mathrm{H}]^{-}$} & 97.07 & C-diet \\
\hline LysoPC $(14: 0)^{\mathrm{I}}$ & 7.12 & 512.2960 & {$[\mathrm{M}+\mathrm{FA}-\mathrm{H}]^{-}$} & $\begin{array}{l}452.30 \\
227.20\end{array}$ & C-diet \\
\hline $\operatorname{PC}(33: 1)^{\mathrm{I}}$ & 8.14 & 790.5627 & {$[\mathrm{M}+\mathrm{FA}-\mathrm{H}]^{-}$} & 184.08 & C-diet \\
\hline $\mathrm{PC}(31: 0)^{\mathrm{I}}$ & 8.17 & 720.5657 & {$[\mathrm{M}+\mathrm{H}]^{+}$} & 184.08 & C-diet \\
\hline $\mathrm{PC}(33: 1)^{\mathrm{I}}$ & 8.25 & $\begin{array}{l}790.5631 \\
746.5730\end{array}$ & $\begin{array}{l}{[\mathrm{M}+\mathrm{FA}-\mathrm{H}]^{-}} \\
{[\mathrm{M}+\mathrm{H}]^{+}}\end{array}$ & 184.08 & C-diet \\
\hline$P C(33: 0)^{I}$ & 8.39 & 748.5876 & {$[\mathrm{M}+\mathrm{H}]^{+}$} & 184.08 & C-diet \\
\hline
\end{tabular}

I-IV: level of identification: I, confirmed with analytical standard; II, putatively annotated, based on spectral similarity to a compound; III, putatively characterized, based on spectral similarity to a chemical class; IV, unknown

$\mathrm{m} / \mathrm{z}$ mass to charge ratio, $R T$ retention time, $P C$ phosphatidylcholine, $P C(P)$ phosphatidylcholine plasmalogen

*Expected isomers

diet groups are forming two major clusters that show no or a very weak correlation. Furthermore, within the clusters, a mutual correlation is found in accordance with the PCA model (Fig. 1). Besides the two major clusters, there are several small sub-clusters; the phospholipids with lower levels in the Ex-diet group all showed a strong correlation with each other, especially two isomers of PC(33:1) (m/z 790.563, RT 8.14 and 8.25) and an unidentified phospholipid (m/z 776.5638, RT 8.13), but also two other unidentified metabolites, $\mathrm{m} / \mathrm{z} 880.6077$ and $\mathrm{m} / \mathrm{z} 832.6107$. Furthermore, C9:0-carnitine correlates with an unknown compound (m/z 155.0677, RT 6.28). Moreover, the two carnitines show a moderate inverse correlation, confirming their associations with the different diet groups.
The serum metabolites most strongly separating the two diet groups are listed in Table 2 . The associations between PC1 and PC2 scores and the clinical outcomes are shown in Table 3. Total cholesterol, LDL-cholesterol, and TG levels are all significantly correlated with PC1 scores. PC1 is dominated by the odd-numbered PCs, e.g. PC(31:0), PC(33:0), and PC(33:1). Furthermore, total cholesterol and LDL-cholesterol levels are inversely correlated with PC2, represented by the two PC(P-38:4) plasmalogens and by C8:1-carnitine. Moreover, HDL-cholesterol levels correlated negatively with PC2. No associations were

\section{Associations between diet-specific metabolic profiles and cardiometabolic risk factors}




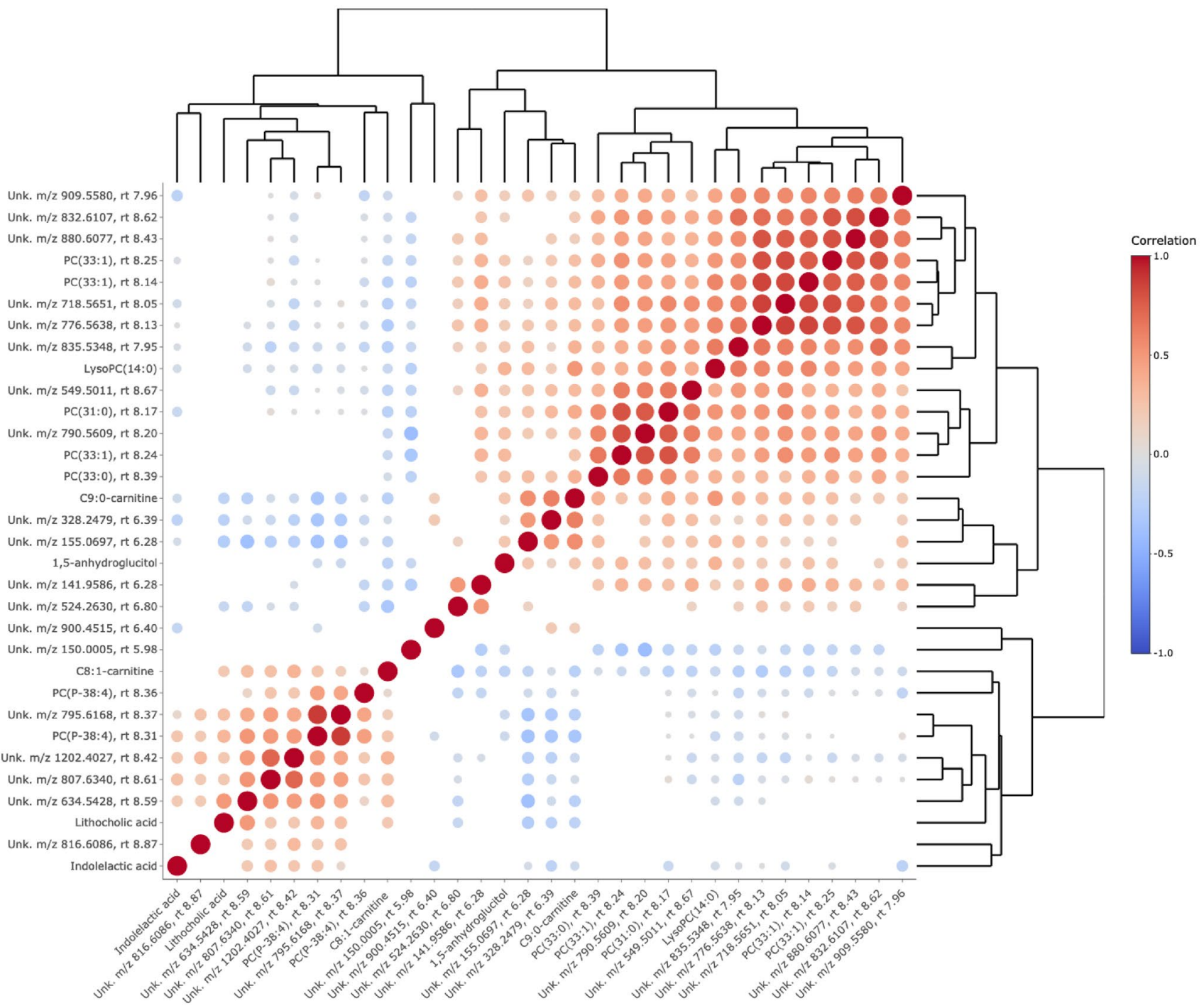

Fig. 2 Hierarchical clustering analysis of the serum metabolites differentiating the Ex-diet diet group from the C-diet group diet based on Spearman correlation coefficients; only significant correlations $(p<0.05)$ are depicted. Unk unknown, $P C(P)$ phosphatidylcholine plasmalogen, $P C$ phosphatidylcholine, $\mathrm{m} / \mathrm{z}$ mass to charge ratio, $R T$ retention time

the subjects from the two groups. This demonstrates that relatively modest alterations in the dietary fat composition of commonly used food items can be detected in the serum metabolome. Furthermore, it also confirms the compliance of the participants over the 8-week intervention, as the metabolites that were lowered in the Ex-diet group reflected food items from the $\mathrm{C}$-diet, while the metabolites that were increased originated from the food items of the Ex-diet group.

In the present study, PUFAs incorporated in PC plasmalogens were characterizing the Ex-diet group. The observed PUFA plasmalogens may be a consequence of the increased dietary intake of especially $n$-6 PUFA from the Ex-diet food items containing rapeseed and sunflower oil. The effect of the Ex-diet food items was also found in the original
The untargeted metabolic profiles of serum samples from this 8-week randomized controlled trial with similar foods containing mainly $n-6$ PUFA vs. SFA clearly differentiated 
Table 3 Association between clinical outcomes and PCA scores reflecting the diet-related serum metabolites

\begin{tabular}{lll}
\hline Clinical measurements & PC & $\mathrm{r}(95 \% \mathrm{CI})$ \\
\hline LDL-cholesterol, mmol/L & PC1 & $0.2599(0.669 ; 0.4342)^{*}$ \\
& PC2 & $-0.2524(-0.4277 ;-0.5895)^{*}$ \\
TG, mmol/L & PC1 & $0.2758(0.0819 ; 0.4497)^{*}$ \\
Total cholesterol, mmol/L & PC2 1 & $-0.2090(-0.4575 ;-0.0959)^{*}$ \\
& PC2 & $-0.2869(-0.4575 ;-0.0959)^{*}$ \\
HDL-cholesterol, mmol/L & PC1 & $0.0065(-0.1902 ; 0.2027)$ \\
& PC2 & $-0.2177(-0.3971 ;-0.0222)^{*}$ \\
Glucose, mmol/L & PC1 & $0.0582(-0.1408 ; 0.2527)$ \\
Insulin, pmol/L & PC2 & $0.0862(-0.1131 ; 0.2789)$ \\
& PC1 & $0.1232(-0.0750 ; 0.3121)$ \\
& PC2 & $-0.0916(-0.2830 ; 0.1067)$ \\
\hline
\end{tabular}

$L D L$ low-density lipoprotein, $T G$ triglyceride, $H D L$ high-density lipoprotein, $P C$ Principal component, $r$ correlation coefficient, 95\%CI $95 \%$ confidence interval

$* p<0.05$

publication of this study where increased arachidonic acid (AA) and linoleic acid (LA) concentrations were detected in serum from the Ex-diet group compared to the C-diet group after the intervention period [15]. Also contributing to the increase of PUFA plasmalogens, AA may have been synthesized from LA by desaturation and elongation enzymes [35]. Additionally, a high intake of LA has previously been inversely associated with total and CVD mortality [36]. Independent of other dietary factors, increased LA consumption has also been associated with reduced risk of CHD events and mortality in a meta-analysis of cohort studies [37].

Another study focusing on the implementation of a Nordic diet has also observed higher levels of plasmalogens [12]. Unsaturated plasmalogens have antioxidant (more precisely, radical scavenging) properties due to their vinylether moiety, which is preferentially oxidized when exposed to various free radicals and singlet oxygen [38]. Increased lipid oxidation has been linked to decreased plasmalogen levels, and associated with disease progression in CVD [39]. Furthermore, plasmalogens have also been proposed to have an important role in cholesterol homeostasis [40]. In this study, we observed that HDL cholesterol levels were inversely associated with PC2 scores (mainly explained by plasmalogens), but also that $\mathrm{PC} 2$ scores were inversely associated with LDL cholesterol, total cholesterol, and TG levels. These correlations suggest that even small alterations in the dietary lipid composition associate with cholesterol levels although we could not find an indication of a causal relationship using mediation analysis in this relatively small study.

Using NMR spectrometry we have previously shown that total PC was reduced in the Ex-diet group compared to the
C-diet group [16]. By using untargeted metabolomics we have been able to identify more specifically the different PCs characterized by the different diet groups. Several of the metabolites characterizing the $\mathrm{C}$-diet group in contrast to the Ex-diet group were the odd-numbered phospholipids, i.e. $\mathrm{PC}(31: 0)$ and $\mathrm{PC}(33: 1)$, potentially containing either pentadecanoic (C15:0) or heptadecanoic (C17:0) acids. These odd-numbered PCs are all strongly sub-clustered, indicating that they might originate from the same dietary components. FAs with odd-numbered chains are likely to originate from dairy products [41]. The lower concentrations of these phospholipids after altering the fat composition confirm the compliance of the participants in the Ex-diet group.

The SYSDIET study also reported reduced levels of (C15:0) in phospholipids in serum for the HND group compared to an average Nordic diet (AND) rich in dairy products [10]. Circulating phospholipids containing pentadecanoic or heptadecanoic acids have been associated with non-CVD mortality, but not with CVD and stroke mortality in an observational study [42]. The correlation analysis between the clinical outcomes from the original publication of the study and the diet-specific metabolic profiles show positive associations between PC1 scores (higher for the C-diet group) and LDL-cholesterol, total cholesterol, and TG. In contrast, no correlation between PC1 scores and HDL-cholesterol was observed. However, the mediation analysis could not explain the changes in total cholesterol, TG, or LDL-cholesterol levels through the altered metabolomes (Table 8SI). This could indicate that other factors not covered by the current serum metabolome may cause the observed changes in the blood lipids.

In the publication of the primary endpoints of this study, a reduction in the level of C14:0 in the plasma lipid concentration was found [15]. In accordance, lower levels of lysoPC(14:0) were observed in the Ex-diet group compared to the C-diet group in this untargeted metabolomics study. LysoPCs are key components in oxidized-LDL and play an important role in the progression of atherosclerosis [43]. Moreover, lysoPCs have been implied as lipid intermediates linking SFA to insulin resistance [44]. A previous study reports lysoPC(14:0) together with decanoylcarnitine (C10:0-carnitine) as a potential predictor for future diabetes [45]. However, we found no association of these markers with glucose- or insulin responses.

The unsaturated medium-chain 2-octenoyl (C8:1-carnitine) was found at higher levels in the Ex-diet group, whereas the saturated nonanoylcarnitine (C9:0-carnitine) was found at lower levels. Like this current sub-study, a previously targeted metabolomics sub-study reported that saturated acylcarnitines (ACs), such as palmitoylcarnitine and myristoylcarnitine, were reduced in the Ex-diet group compared to the C-diet group [16]. Furthermore, mediumchain ACs have also been independently associated with 
mortality from myocardial infarction and with the risk of stroke and total CVD in subjects at high risk [46, 47].

In addition to the phospholipids and ACs, other compounds related to the alteration of the lipid composition of the diet were identified. One of the more interesting ones is the lower levels of 1,5-AG in the Ex-diet group. The 1,5-AG is a monosaccharide derived primarily from dietary sources and supposed to be found in constant concentrations in the blood in subjects having normal glycaemic status [48]. The 1,5-AG concentration is balanced by urinary excretion, and the kidneys reabsorb nearly all. However, reabsorption is competitively inhibited by glucose, meaning that $1,5-\mathrm{AG}$ is not excreted in urine before glucose surpasses the renal threshold for glycosuria (generally around $180 \mathrm{mg} / \mathrm{dl}$ ) that then leads to a rapid decrease in serum levels $[49,50]$. Hence, poor glycaemic control is associated with low 1,5AG serum levels.

It is difficult to explain the observed lower level of 1,5AG in the Ex-diet group compared to the C-diet group of this study, as it has been found that consuming vegetable oils high in LA (like the Ex-diet group) improves insulin sensitivity contributing to a more stable glucose level [51]. A recent metabolomics study with HND vs. average Danish diet also identified 1,5-AG at lower levels in the intervention group in relation to weight loss [52]. Only a minor increase in weight was observed for the C-diet group during the intervention; however, no change was observed in the Ex-diet group. Furthermore, no change in either insulin, glucose, or HbA1c levels was observed in the current study and neither glucose nor insulin showed any correlation to the dietspecific metabolic profiles. This indicates that the alteration of lipids in the diet was not sufficient to change glycaemia, but still sufficient to affect the levels of 1,5-AG. This may suggest that the 1,5-AG levels might have been affected by other mechanisms than the fat alteration, possibly a change in dietary exposure to the compound or other compounds than glucose affecting its reabsorption.

LCA is one of the two major secondary bile acids in humans formed in the large intestine. Bile acids are the end product of cholesterol metabolism and are synthesized in the liver. An increase in total bile acids has earlier been found as an effect of a diet rich in PUFA [53]. The higher levels of LCA in the Ex-diet group of this study are in line with this finding and of an increase of total bile acid concentrations in serum in the current study by metabolic profiling [16].

Lastly, serum levels of ILA - a tryptophan metabolite formed by proteolysis in the colon [54] - were higher in the Ex-diet compared to the C-diet group. Contradictory, another recent metabolomics study found ILA, together with other metabolites, to be characteristics of an average Danish diet [55]. However, due to the lack of microbial data further studies are needed to fully understand the relationship of dietary factors with microbial metabolites.
In conclusion, by utilizing an untargeted metabolomics approach it was possible to differentiate the metabolome based on a diet rich in saturated fat from the metabolome based on a diet rich in unsaturated fat. The metabolomes of the Ex-diet and the $\mathrm{C}$-diet groups were reflected by several different metabolites, mainly found here as polar lipids. Previously reported changes in cholesterol and TG were associated with these contrasting metabolites; however, mediation analysis implied that the clinical changes were not explained by the altered serum metabolome. Markers related to glycaemia were not associated with the polar lipid metabolome recorded in the current study. Further studies are needed to identify metabolites that could explain the clinical outcomes of diets rich in saturated or unsaturated fats.

Supplementary Information The online version contains supplementary material available at https://doi.org/10.1007/s00394-021-02796-6.

Acknowledgements The authors thank the NoMa team at the Oslo and Akershus University College of Applied Sciences, Department of Health, Nutrition and Management, and at the University of Oslo, Department for Nutrition, Oslo, Norway. They thank also Sarah Fleischer Ben Soltane, Cecilie Fryland Appeldorff, and Cristian de Gobba, all from the University of Copenhagen, Department of Nutrition, Exercise and Sports, Copenhagen, Denmark for excellent technical support.

Author contributions SMU and $\mathrm{KBH}$ designed the intervention and were principal investigators of the trial; LOD was responsible for the metabolomics profiling, and KP and GG conducted the metabolomics data analysis; SMU and KBH provided essential materials; KP, ODRH, and GG performed statistical analysis; KP wrote the paper; SMU and LOD had primary responsibility for final content; all the authors approved the final manuscript.

Funding This study was funded by the University of Oslo, Norway, the Throne-Holst Foundation for Nutrition Research, Oslo, Norway and Mills DA and by a Semper Ardens grant on biomarker development from the Carlsberg Foundation for LOD.

Availability of data and material All data, materials, and software applications support published claims and comply with field standards.

Code availability Not applicable.

\section{Declarations}

Conflict of interest Dr. Ulven and Dr. Holven received research grants from Mills DA to partially fund the study. None owns any stocks or serves as an advisory board in the company. The authors declare they have no conflict of interest.

Ethical approval All procedures performed in this study involving the human participants followed the ethical standards of the Regional Ethics Committee for the Medical Research in South East Norway (approval number 24248) and the 1964 Helsinki declaration and its later amendments or comparable ethical standards.

Consent to participate Consent to participate in research has been obtained. 
Consent for publication Consent to publish has been obtained.

Open Access This article is licensed under a Creative Commons Attribution 4.0 International License, which permits use, sharing, adaptation, distribution and reproduction in any medium or format, as long as you give appropriate credit to the original author(s) and the source, provide a link to the Creative Commons licence, and indicate if changes were made. The images or other third party material in this article are included in the article's Creative Commons licence, unless indicated otherwise in a credit line to the material. If material is not included in the article's Creative Commons licence and your intended use is not permitted by statutory regulation or exceeds the permitted use, you will need to obtain permission directly from the copyright holder. To view a copy of this licence, visit http://creativecommons.org/licenses/by/4.0/.

\section{References}

1. Daneshzad E, Emami S, Darooghegi Mofrad M et al (2018) Association of modified Nordic diet with cardiovascular risk factors among type 2 diabetes patients: a cross-sectional study. J Cardiovasc Thorac Res 10:153-161. https://doi.org/10.15171/jcvtr.2018. 25

2. Adamsson V, Reumark A, Fredriksson IB et al (2011) Effects of a healthy Nordic diet on cardiovascular risk factors in hypercholesterolaemic subjects: a randomized controlled trial (NORDIET). J Intern Med 269:150-159. https://doi.org/10.1111/j.1365-2796. 2010.02290.x

3. Micha R, Mozaffarian D (2010) Saturated fat and cardiometabolic risk factors, coronary heart disease, stroke, and diabetes: a fresh look at the evidence. Lipids 45:893-905. https://doi.org/10.1007/ s11745-010-3393-4

4. Jakobsen MU, O'Reilly EJ, Heitmann BL et al (2009) Major types of dietary fat and risk of coronary heart disease: a pooled analysis of 11 cohort studies. Am J Clin Nutr. https://doi.org/10.3945/ajen. 2008.27124

5. Mozaffarian D, Micha R, Wallace S (2010) Effects on coronary heart disease of increasing polyunsaturated fat in place of saturated fat: a systematic review and meta-analysis of randomized controlled trials. PLoS Med. https://doi.org/10.1371/journal. pmed.1000252

6. Thorning TK, Bertram HC, Bonjour JP et al (2017) Whole dairy matrix or single nutrients in assessment of health effects: current evidence and knowledge gaps. Am J Clin Nutr 105:1033-1045. https://doi.org/10.3945/ajcn.116.151548

7. Astrup A, Bertram HC, Bonjour J-P et al (2019) WHO draft guidelines on dietary saturated and trans fatty acids: time for a new approach? BMJ 4137:14137. https://doi.org/10.1136/bmj. 14137

8. Adamsson V, Reumark A, Cederholm T et al (2012) What is a healthy Nordic diet? Foods and nutrients in the NORDIET study. Food Nutr Res 56:1-8. https://doi.org/10.3402/fnr.v56i0.18189

9. Mithril C, Dragsted LO, Meyer C et al (2012) Dietary composition and nutrient content of the new Nordic diet. Public Health Nutr 16:777-785. https://doi.org/10.1017/S1368980012004521

10. Uusitupa M, Hermansen K, Savolainen MJ et al (2013) Effects of an isocaloric healthy Nordic diet on insulin sensitivity, lipid profile and inflammation markers in metabolic syndrome - a randomized study (SYSDIET). J Intern Med 274:52-66. https://doi. org/10.1111/joim. 12044

11. Berild A, Holven KB, Ulven SM (2017) Recommended Nordic diet and risk markers for cardiovascular disease. Tidsskr Nor Laegeforen 137:721-726. https://doi.org/10.4045/tidsskr.16.0243
12. Lankinen M, Schwab U, Kolehmainen M et al (2015) A healthy Nordic diet alters the plasma lipidomic profile in adults with features of metabolic syndrome in a multicenter randomized dietary intervention. J Nutr 146:662-672. https://doi.org/10.3945/jn.115. 220459

13. Marklund M, Hermansen K, Schwab U et al (2014) A dietary biomarker approach captures compliance and cardiometabolic effects of a healthy Nordic diet in individuals with metabolic syndrome. J Nutr 144:1642-1649. https://doi.org/10.3945/jn.114.193771

14. Poulsen SK, Due A, Jordy AB et al (2014) Health effect of the new Nordic diet in adults with increased waist circumference: a 6-mo randomized controlled trial. Am J Clin Nutr 99:35-45. https://doi. org/10.3945/ajen.113.069393

15. Ulven SM, Leder L, Elind E et al (2016) Exchanging a few commercial, regularly consumed food items with improved fat quality reduces total cholesterol and LDL-cholesterol: a double-blind, randomised controlled trial. Br J Nutr 116:1383-1393. https:// doi.org/10.1017/S0007114516003445

16. Ulven SM, Christensen JJ, Nygård O et al (2019) Using metabolic profiling and gene expression analyses to explore molecular effects of replacing saturated fat with polyunsaturated fat-a randomized controlled dietary intervention study. Am J Clin Nutr 109:1239-1250. https://doi.org/10.1093/ajcn/nqy356

17. Brennan L (2013) Metabolomics in nutrition research: current status and perspectives. Biochem Soc Trans 41:670-673. https:// doi.org/10.1042/bst20120350

18. McGarrah RW, Crown SB, Zhang GF et al (2018) Cardiovascular metabolomics. Circ Res 122:1238-1258. https://doi.org/10.1161/ CIRCRESAHA.117.311002

19. Andersen M-BS, Rinnan Å, Manach C et al (2014) Untargeted metabolomics as a screening tool for estimating compliance to a dietary pattern. J Proteome Res 13:1405-1418. https://doi.org/10. 1021/pr400964s

20. Li J, Guasch-Ferré M, Chung W et al (2020) The Mediterranean diet, plasma metabolome, and cardiovascular disease risk. Eur Heart J 41:2645-2656. https://doi.org/10.1093/eurheartj/ehaa209

21. Meslier V, Laiola M, Roager HM et al (2020) Mediterranean diet intervention in overweight and obese subjects lowers plasma cholesterol and causes changes in the gut microbiome and metabolome independently of energy intake. Gut 69:1258-1268. https:// doi.org/10.1136/gutjnl-2019-320438

22. Gürdeniz G, Kristensen M, Skov T, Dragsted LO (2012) The effect of LC-MS data preprocessing methods on the selection of plasma biomarkers in fed vs. fasted rats. Metabolites 2:77-99. https://doi.org/10.3390/metabo2010077

23. Andersen M-BS, Reinbach HC, Rinnan $\AA$ et al (2013) Discovery of exposure markers in urine for Brassica-containing meals served with different protein sources by UPLC-qTOF-MS untargeted metabolomics. Metabolomics 9:984-997. https://doi.org/ 10.1007/s11306-013-0522-0

24. Pluskal T, Castillo S, Villar-Briones A, Orešič M (2010) MZmine 2: modular framework for processing, visualizing, and analyzing mass spectrometry-based molecular profile data. BMC Bioinform. https://doi.org/10.1186/1471-2105-11-395

25. Szymańska E, Saccenti E, Smilde AK, Westerhuis JA (2012) Double-check: validation of diagnostic statistics for PLS-DA models in metabolomics studies. Metabolomics 8:3-16. https://doi.org/ 10.1007/s11306-011-0330-3

26. R Core Development Team A Language and Environment for Statistical Computing. In: R. https://www.r-project.org

27. Tingley D, Yamamoto T, Keele L, Kosuke I (2014) Mediation: $\mathrm{R}$ package for causal mediation analysis. R Packag Version 424. https://doi.org/10.18637/jss.v059.i05

28. Smith CA, O'Maille G, Want EJ et al (2005) METLIN: a metabolite mass spectral database. Ther Drug Monit 27:747-751 
29. Wishart DS, Feunang YD, Marcu A et al (2018) HMDB 4.0: the human metabolome database for 2018. Nucleic Acids Res 46:D608-D617. https://doi.org/10.1093/nar/gkx1089

30. Djoumbou-Feunang Y, Pon A, Karu N et al (2019) CFM-ID 3.0: significantly improved ESI-MS/MS prediction and compound identification. Metabolites 9:72. https://doi.org/10.3390/metab o9040072

31. Dührkop K, Fleischauer M, Ludwig M et al (2019) SIRIUS 4: a rapid tool for turning tandem mass spectra into metabolite structure information. Nat Methods 16:299-302. https://doi.org/10. 1038/s41592-019-0344-8

32. Sud M, Fahy E, Cotter D et al (2007) LMSD: LIPID MAPS structure database. Nucleic Acids Res 35:527-532. https://doi.org/10. 1093/nar/gkl838

33. Gürdeniz G, Rago D, Bendsen NT et al (2013) Effect of trans fatty acid intake on LC-MS and NMR plasma profiles. PLoS ONE 8:e69589. https://doi.org/10.1371/journal.pone.0069589

34. Sumner LW, Reily MD, Higashi R et al (2007) Proposed minimum reporting standards for chemical analysis. Metabolomics 3:211-221. https://doi.org/10.1007/s11306-007-0082-2

35. Horrobin F (1993) Fatty acid metabolism in health the role of $\Delta 6$-desaturase. Am J Clin Nutr 57:732-737. https://doi.org/10. 1093/ajen/57.5.732S

36. Wu JHY, Lemaitre RN, King IB et al (2014) Circulating omega-6 polyunsaturated fatty acids and total and cause-specific mortality. Circulation 130:1245-1253. https://doi.org/10.1161/circulatio naha.114.011590

37. Farvid MS, Ding M, Pan A et al (2014) Dietary linoleic acid and risk of coronary heart disease: a systematic review and meta-analysis of prospective cohort studies. Circulation 130:1568-1578. https://doi.org/10.1161/CIRCULATIONAHA.114.010236

38. Broniec A, Klosinski R, Pawlak A et al (2011) Interaction of plasmalogens and their diacyl analogs with singlet oxygen in selected model systems. Free Radic Biol Med 50:892-898. https://doi.org/ 10.1016/j.freeradbiomed.2011.01.002.Interaction

39. Braverman NE, Moser AB (2012) Functions of plasmalogen lipids in health and disease. Biochim Biophys Acta - Mol Basis Dis 1822:1442-1452. https://doi.org/10.1016/j.bbadis.2012.05.008

40. Honsho M, Fujiki Y (2017) Plasmalogen homeostasis - regulation of plasmalogen biosynthesis and its physiological consequence in mammals. FEBS Lett 591:2720-2729. https://doi.org/10.1002/ 1873-3468.12743

41. Pfeuffer M, Jaudszus A (2018) Pentadecanoic and heptadecanoic acids: multifaceted odd-chain fatty acids. Adv Nutr. https://doi. org/10.3945/an.115.011387

42. De Oliveira Otto MC, Lemaitre RN, Song X et al (2018) Serial measures of circulating biomarkers of dairy fat and total and cause-specific mortality in older adults: the cardiovascular health study. Am J Clin Nutr 108:476-484. https://doi.org/10.1093/ajen/ nqy 117
43. Li R-S (2015) Lysophospholipids and their G protein-coupled receptors in atherosclerosis. Front Biosci 21:70-88. https://doi. org/10.2741/4377

44. Han MS, Lim Y-M, Quan W et al (2011) Lysophosphatidylcholine as an effector of fatty acid-induced insulin resistance. J Lipid Res 52:1234-1246. https://doi.org/10.1194/jlr.m014787

45. Ha CY, Kim JY, Paik JK et al (2012) The association of specific metabolites of lipid metabolism with markers of oxidative stress, inflammation and arterial stiffness in men with newly diagnosed type 2 diabetes. Clin Endocrinol (Oxf) 76:674-682. https://doi. org/10.1111/j.1365-2265.2011.04244.x

46. Guasch-Ferré M, Zheng Y, Ruiz-Canela M et al (2016) Plasma acylcarnitines and risk of cardiovascular disease: effect of Mediterranean diet interventions. Am J Clin Nutr 103:1408-1416. https://doi.org/10.1093/ajcn/57.5.732S

47. Shah SH, Sun JL, Stevens RD et al (2012) Baseline metabolomic profiles predict cardiovascular events in patients at risk for coronary artery disease. Am Heart J 163:844-850.e1. https://doi.org/ 10.1016/j.ahj.2012.02.005

48. Ikeda N, Hara H, Hiroi Y, Nakamura M (2016) Impact of serum 1,5-anhydro-d-glucitol level on prediction of major adverse cardiac and cerebrovascular events in non-diabetic patients without coronary artery disease. Atherosclerosis 253:1-6. https://doi.org/ 10.1016/j.atherosclerosis.2016.08.016

49. Dungan KM (2008) 1,5-Anhydroglucitol (GlycoMark ${ }^{\mathrm{TM}}$ ) as a marker of short-term glycemic control and glycemic excursions. Expert Rev Mol Diagn 8:9-19. https://doi.org/10.1586/14737 159.8.1.9

50. Ying L, Ma X, Yin J et al (2018) The metabolism and transport of 1,5-anhydroglucitol in cells. Acta Diabetol 55:279-286. https:// doi.org/10.1007/s00592-017-1093-8

51. Hayes J, Benson G (2016) What the latest evidence tells us about fat and cardiovascular health. Diabetes Spectr 29:171-175. https:// doi.org/10.2337/diaspect.29.3.171

52. Khakimov B, Poulsen SK, Savorani F et al (2016) New nordic diet versus average Danish diet: a randomized controlled trial revealed healthy long-term effects of the new Nordic diet by GC-MS blood plasma metabolomics. J Proteome Res 15:1939-1954. https://doi. org/10.1021/acs.jproteome.6b00109

53. Bosaeus IG, Andersson HB (1987) Short-term effect of two cholesterol-lowering diets on sterol excretion in ileostomy patients. Am J Clin Nutr 45:54-59. https://doi.org/10.1093/ajcn/45.1.54

54. Roager HM, Licht TR (2018) Microbial tryptophan catabolites in health and disease. Nat Commun 9:1-10. https://doi.org/10.1038/ s41467-018-05470-4

55. Acar E, Gürdeniz G, Khakimov B et al (2019) Biomarkers of individual foods, and separation of diets using untargeted LC-MSbased plasma metabolomics in a randomized controlled trial. Mol Nutr Food Res 63:1-10. https://doi.org/10.1002/mnfr.201800215 